
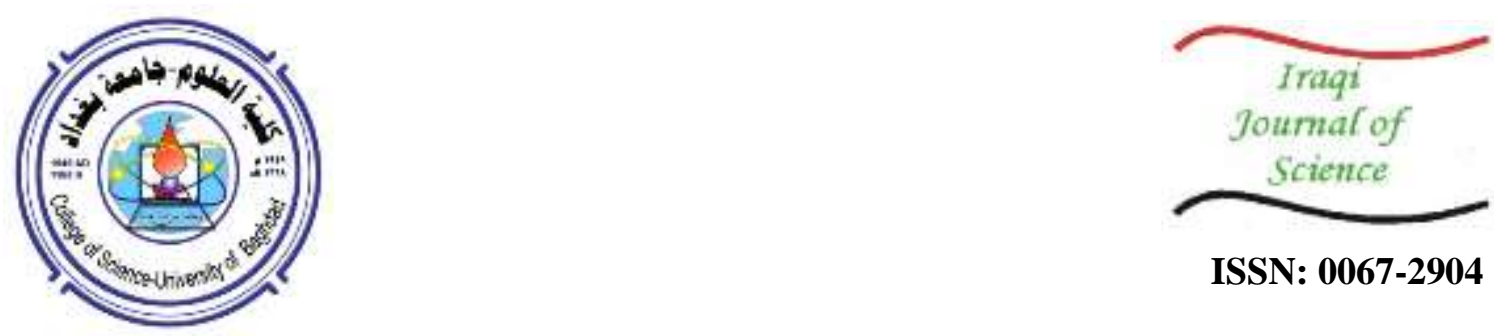

ISSN: 0067-2904

\title{
I-Nearly Prime Submodules
}

\author{
Adwia J. Abdul-AlKalik ${ }^{1}$, Nuhad S. Al-Mothafar ${ }^{2}$
}

${ }^{1}$ Republic of Iraq, Ministry of Education, Directorate General of Education In Diyala ${ }^{2}$ Department of Mathematics, College of Science, University of Baghdad, Iraq

\begin{abstract}
:
Let $\mathrm{R}$ be a commutative ring with identity, and $I$ a fixed ideal of $R$ and $M$ be an unitary $R$-module. In this paper we introduce and study the concept of $I$-nearly prime submodules as genrealizations of nearly prime and we investigate some properties of this class of submodules. Also, some characterizations of $I$-nearly prime submodules will be given.
\end{abstract}

Received: 7/4/ $2019 \quad$ Accepted: 18/6/2019

Keywords: Prime submodules, nearly prime submodules, $I$-prime submodules.

\section{Introduction}

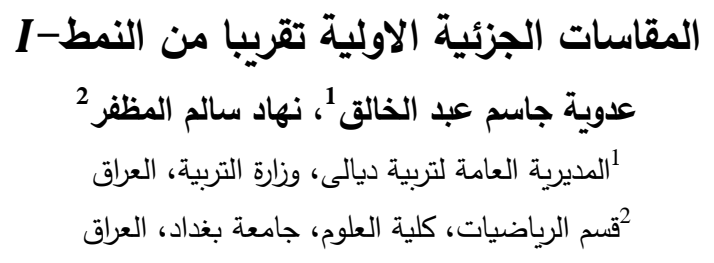

لتكن R حلقة ابدالية ذات عنصرمحايد، وليكن I مثالي من R ، M مقاسا احاديا معرفا على الحلقة R.

قدمنا ودرسنا في هذا البحث المفهوم :المقاسات الجزئية الاولية تقريبا من النمط-I كاعمام للمقاسات الجزئية

الاولية تقريبا ـ لقد درسنا واعطينا بعض خواص ومميزات هذا النوع من المقاسات الجزئية.

Throughout, $R$ represents an associative ring with nonzero identity and $I$ a fixed ideal of $R$. A proper submodule $N$ of $M$ is called a prime submodule if whenever $r \in R$ and $x \in M$ with $r x \in N$ implies that $r \in[N: M]$ or $x \in N$, [1]. One of generalization of this concept was studied as nearly prime, [2]. A proper submodule $N$ of $M$ is called nearly prime submodule if whenever $r \in$ $R, x \in M$ and $r x \in N$ implies that either $x \in N+J(M)$ or $r \in[N+J(M): M]$. Previuos work [3] and [4] introduced the notions $I$ - prime and $I$ - primary submodules. A proper submodule $\mathrm{N}$ of $\mathrm{M}$ is called I- prime submodule of $\mathrm{M}$ if $\mathrm{rx} \in \mathrm{N}$ - IN for all $r \in R, x \in M$ implies that either $r \in[N: M]$ or $x \in N$. A proper submodule $\mathrm{N}$ of $\mathrm{M}$ is called I- primary submodule of $\mathrm{M}$ if $\mathrm{rx} \in \mathrm{N}-\mathrm{IN}$ for all $r \in R, x \in M$ implies that either $r \in \sqrt{[N: M]}$ or $x \in N$. In this paper, we define and study $I$ - nearly prime submodules which are generalizations of prime submodules and nearly prime submodules to $I$ - nearly prime submodules. A proper submodule $N$ of $M$ is called $I$-nearly prime submodule if $\mathrm{rx} \in \mathrm{N}-$ IN for all $r \in R, x \in M$ implies that either $r \in[N+J(M): M]$ or $x \in N+J(M)$, where $J(M)$ is the intersection of all maximal submodule of $M$. Here is a brief summary of the paper. In Theorem 1.4 we show that $N$ is $I$-nearly prime submodule in $M$ if and only if for any ideal $J$ of $R$ and submodule $K$ of $M$ such that $J K \subsetneq N-I N$, we have $\mathrm{K} \subsetneq \mathrm{N}+\mathrm{J}(\mathrm{M})$ or $J \subsetneq[N+J(M): M]$. In Proposition 1.6 we show that if $N$ is an $I$ - nearly prime in $M$ and $[N: M] N \nsubseteq I N$, then $N$ is a nearly prime in $M$. In Theorem 1.8 we show that if $N$ is a submodule of an $R$-module $M$ then the following statements are

*Email: adwiaj@yahoo.com 
equivalent: (1) $N$ is an $I$-nearly prime in $M$. (2) For $r \in R \backslash[N+J(M): M],[N: r]=[N+J(M)] \cup$ $[I N: r]$. (3) For $r \in R \backslash[N+J(M): M],[N: r]=N+J(M)$ or $[N: r]=[I N: r]$.

\section{Main result}

Definition 1.1: A proper submodule $N$ of an $R$-moadule $M$ is called $I$-nearly prime submodule if and only if whenever $r \in R, x \in M$ and $r x \in N-I N$ implies that either $x \in N+J(M)$ or $r \in$ $[N+J(M): M]$, where $I$ is an ideal of $R$ and $J(M)$ is the Jacobson radical of $M$.

For examples: 1 - Consider the ring of integers $\mathrm{Z}$ and the Z-module Z12. Take $\mathrm{I}=4 \mathrm{Z}$ as an ideal of $\mathrm{Z}$ and $\mathrm{N}=(\overline{4})$ be a submodule of $\mathrm{Z12}$ generated by

4. Then $\mathrm{N}$ is an I-nearly prime submodule of Z12 since $\mathrm{N}-\mathrm{IN}=(\overline{4})-4 \mathrm{Z} .(\overline{4})=$

$(\overline{4})-(\overline{4})=\varnothing$. On the other side, $\mathrm{N}$ is not a prime submodule since $\overline{4}=2 . \overline{2} \in \mathrm{N}$ but not $\overline{2} \in \mathrm{N}$ nor $2 \in$ [N:Z12 ].

2- Let $\mathrm{N}=(\overline{0})$ is an I-nearly prime submodule of $\mathrm{Z} 6$ as a $\mathrm{Z}$-module since if $\mathrm{I}=(0)$ is taken as an ideal of $\mathrm{Z}$, then $\mathrm{N}-\mathrm{IN}=(\overline{0})-(0) .(\overline{0})=\varnothing$. On the other side, $\mathrm{N}$ is not a nearly prime submodule, see [2].

3- Consider the ring of integers $\mathrm{Z}$ and the $\mathrm{Z}$-module $\mathrm{Z} 40$ and $\mathrm{N}=(\overline{8})$. Take

$\mathrm{I}=[\mathrm{N}: \mathrm{M}]=8 \mathrm{Z}$ as an ideal of $\mathrm{Z}$, then $[\mathrm{N}: \mathrm{M}] \mathrm{N}=\mathrm{N}$. Then $\mathrm{N}$ is an I-nearly prime submodule of $\mathrm{Z} 12$ since $\mathrm{N}-\mathrm{IN}=(\overline{8})-8 \mathrm{Z} .(\overline{8})=\emptyset$. On the other side, if $\mathrm{I}=(0)$ is taken as an ideal of $\mathrm{Z}$, then $[\mathrm{N}: \mathrm{M}] \mathrm{N}=(0)$, then $\mathrm{N}$ is not an I-nearly prime submodule since $\overline{8}=4 . \overline{2} \in \mathrm{N}$ but not $\overline{2} \in \mathrm{N}$ nor $4 \in[\mathrm{N}: \mathrm{Z} 12]$.

Proposition (1. 2):

1- If $N$ is an $I$ - nearly prime in $M$ and $K$ is a submodule of $M$ with $J(M) \subseteq J(K)$, then $N$ is an $I$ nearly prime submodule of $K$.

2- If $I_{1} \subseteq I_{2}$. Then $N$ is an $I_{1}$ - nearly prime implies $N$ is $I_{2}$ - nearly prime.

Proof. (1): Suppose that $a m \in N-I N$ where $a \in R$ and $m \in K$. Since $N$ is an $I$-nearly prime submodule of $M$, so either $m \in N+J(M)$ or $a \in[N+J(M): M]$. But $J(M) \subseteq J(K)$, so either $m \in N+J(K)$ or $a \in[N+J(K): K]$.Therefore $N$ is an $I$ - nearly prime submodule of $K$.

(2): Let $m \in M$ and $m \in M$ with $a m \in N-I_{2} N$. Since $I_{1} \subseteq I_{2}, N-I_{2} N \subseteq N-I_{1} N$, then $a m \in$ $N-I_{1} N$. But $N$ is an $I_{1}$ - nearly prime. So $a \in[N+J(M): M]$ or $m \in N+J(M)$. Thus $N$ is an $I_{2^{-}}$ nearly prime.

Proposition (1.3): Let $N$ be a submodule of an $R$ - module $M$.

1 - If $N$ is an $I$-nearly prime and $J(M) \subseteq N$, then $N$ is an $I$-prime (and $I$-primary) .

2- If $N$ is a maximal an $I$ - nearly prime submodule of a local $R$-module $M$, then $\mathrm{N}$ is an $I$-prime (and $I$-primary) in $M$.

3- If $\mathrm{N}$ is an $I$ - nearly prime submodule of a semisimple $R$-module $M$, then $N$ is an $I$-prime (and $I$ primary) in $\mathrm{M}$.

Proof. (1).The proof is trivial.

(2). Suppose that $a m \in N-I N$ where $a \in R, m \in M$. Since $N$ is an $I$-nearly prime submodule of $M$, so either $m \in N+J(M)$ or $a \in[N+J(M): M]$. But $M$ be a local and $N$ is a maximal, so $J(M)=$ $N$, [5]. So either $m \in N$ or $a \in[N: M]$.Therefore $N$ is an $I$-prime (and $I$-primary) in $M$.

(3). Suppose that $a m \in N-I N$ where $m \in M$ and $a \in R$. Because $N$ is an $I$-nearly prime submodule of $M$, so either $a \in[N+J(M): M]$ or $m \in N+J(M)$. But $M$ be a semisimple an $R-$ module, so $J(M)=0$, [6]. So either $m \in N$ or $a \in[N: M]$. Hence $N$ is an I-prime (and $I$-primary) in $M$.

The following theorem gives a useful characterization for an $I$-nearly prime submodules.

Theorem (1.4): Let $N$ be a proper submodule of an $R$-module $M$. Then $N$ is an $I$-nearly prime submodule in $M$ if and only if for any ideal $J$ of $R$ and submodule $K$ of $M$ such that $J K \subsetneq N-I N$, we have $\mathrm{K} \subsetneq \mathrm{N}+\mathrm{J}(\mathrm{M})$ or $J \subsetneq[N+J(M): M]$.

Proof. Suppose that $N$ is an $I$-nearly prime in $M$. Let $J K \subsetneq N-I N$ for some ideal $J$ of $R$ and submodule $K$ of $M$. If $J \nsubseteq[N+J(M): M]$ and $K \nsubseteq N+J(M)$, so there exists $\quad r \in J \backslash[N+$ $J(M): M]$ and $x \in K \backslash[N+J(M)]$ such that $r x \in N-I N$.

By assuming that $N$ is an $I$-nearly prime submodule in $M$, either $x \in N+J(M)$ or $r \in[N+J(M): M]$ which is a contradiction. Hence $J \subsetneq[N+J(M): M]$ or $K \subsetneq N+J(M)$.

Conversely supposse that $r m \in N-I N$ where $r \in R, m \in M$. So $(r)(m)=(r m)$

$\subsetneq N-I N$. So, either $(r) \subsetneq[N+J(M): M]$ or $(m) \subsetneq N+J(M)$. Therefore $r \in[N+J(M): M]$ or $m \in N+J(M)$. Thus $N$ is an $I$-nearly prime submodule of $M$. 
Let $R$ be a ring . A subset $S$ of $R$ is called multiplicatively closed subset if $1 \in S$ and $a b \in S$, $\forall a, b \in S$, [7].

Let $R_{S}$ be the set of all fractional $r / s$ where $r \in R$ and $s \in S$ and $M_{S}$ be the set of all farctional $x / s$ where $x \in M$ and $s \in S$. For $x_{1}, x_{2} \in M$ and $s_{1}, s_{2} \in S, x_{1} / s_{1}=x_{2} / s_{2}$ if and only if there exists $t \in S$ such that $t\left(s_{1} x_{1}-s_{2} x_{2}\right)=0$.

So, we can make $M_{s}$ into $R_{s}$-module by setting $x / s+y / t=(t x+s y) / s t$ and $r / t . x / s=$ $r x / t s$ ) for every $x, y \in M$ and $s, t \in S, r \in R$. And $M_{s}$ is the module of fractions. If $N$ is a submodule of $M$, so $N_{S}=\{n / s ; n \in N, s \in S\}$ is a submodule of $M_{S}$, [7].

The quotient and localization of prime submodules are again prime submodules. But in case of $I$ nearly prime submodules, we give a condition under which the quotient and localization become true.

Proposition (1.5): Suppose that $N$ is an $I$-nearly prime in $M$.

1) If $N_{S} \neq M_{S}$ and $(I N) s \subseteq I s N s$. Then $N_{S}$ is an $I_{S}$-nearly prime submodule of an $R_{S}$-module $M_{S}$.

2) If $K \subseteq N$ and $N / K+J(M / K)=N+J(M) / K$, then $N / K$ is an $I$-nearly prime in $M / K$.

Proof (1): For all $r / s \in R_{S}$ and $x / t \in M \mathrm{~s}$, let $r / s . x / t=r x / s t \in N s-I s N s \subseteq N_{S}-(I N) s=$ $(N-I N) s$. Then $r x / s t=m / u$ for $m \in N-I N$ and $u \in S$. So for some $v \in S$, vurx $=$ vstm $\in N-I N$. As $N$ is an $I$-nearly prime submodule, so either vur $\in[N+J(M): M]$ or $x \in N+$ $J(M)$. So ruv $/ \mathrm{suv}=r / s \in[N+J(M): M]_{S}=\left[N_{S}+J\left(M_{S}\right): M_{S}\right]$ or $\frac{x}{t} \in[N+J(M)]_{S}=N_{S}+$ $J\left(M_{S}\right)$ by [8]. Hence $N_{S}$ is an $I_{S}$-nearly prime in $M_{S}$.

(2): Suppose that $m \in M$ and $a \in R$ with $a(m+K)=a m+K \in N / K-I(N / K)$.

Then $a m+K \in[N-I N] / K$. So $a m \in N-I N$. Since $N$ is an $I$-nearly prime submodule of $M$, so either $m \in N+J(M)$ or $a \in[N+J(M): M]$. Therefore $m+K \in N / K+J(M / K)$ or $a \in[N / K+$ $J(M / K): M / K]$. Therefore $N / K$ is an $I$ - nearly prime in $M / K$.

Proposition (1.6): If $N$ is an $I$ - nearly prime in $M$ and $[N: M] N \nsubseteq I N$, then $N$ is a nearly prime in $M$. Proof: We show that $N$ is a nearly prime. Suppose that $a m \in N$ where $m \in M, a \in R$.

If $a m \notin I N$, then $N, I$ - nearly prime gives $m \in N+J(M)$ or $a \in[N+J(M): M]$. So assume that $a m \in I N$. First suppose that $a N \nsubseteq I N$, say an $\notin I N$ where $n \in N$.Then $a(m+n) \in N-I N$, so $a$ $\in[N+J(M): M]$ or $(m+n) \in N+J(M)$. Hence $a \in[N+J(M): M]$ or $m \in N+J(M)$. Now, if $m[N: M] \nsubseteq I N$.So $\exists b \in[N: M]$ such that $m b \notin I N$. So $(a+b) m \in N$. Therefore $m \in N+J(M)$ or $(a+b) \in[N+J(M): M]$. Then $m \in N+J(M)$ or $a \in[N+J(M): M]$. Suppose that $m[N: M] \subseteq$ $I N$. Since $[N: M] N \nsubseteq I N$, there exists $r \in[N: M], x \in N$ with $r x \notin I N$. Then $(a+r)(m+$ $x) \in N-I N$. Then $(a+r) \in[N+J(M): M]$ or $(m+x) \in N+J(M)$. Hence $a \in[N+J(M): M]$ or $m \in N+J(M)$. So $N$ be a nearly prime in $M$.

Corollary (1.7): If $N$ is an 0 - nearly prime in $M$ and $[N: M] N \neq 0$. Then $N$ is a nearly prime in $M$.

In what follows we give some charactrizations for an $I$-nearly prime .

Theorem (1.8): Suppose that $N$ is a submodule of an $R$-module $M$. Then the following statements are equivalent:

(1) $N$ is an $I$-nearly prime in $M$.

(2) For $r \in R \backslash[N+J(M): M],[N: r]=[N+J(M)] \cup[I N: r]$.

(3) For $r \in R \backslash[N+J(M): M]$, $[N: r]=N+J(M)$ or $[N: r]=[I N: r]$.

Proof: (1) $\rightarrow$ (2): Suppose that $N$ is an $I$-nearly prime submodule of $M$ such that $r \notin[N+$ $J(M): M]$. Let $m \in[N: r]$. So $r m \in N$. If $r m \notin I N$, then $m \in N+J(M)$.

Because $N$ is an $I$-nearly prime submodule in $M$. If $r m \in I N$, so $m \in[I N: r]$. Hence $[N: r] \subsetneq[N+$ $J(M)] \cup[I N: r]$. Now since $I N \subsetneq N$, the other inclusion is hold.

(2) $\rightarrow$ (3): Because $[N: r]$ is a submodule of $M$, so it is clear.

$(3) \rightarrow(1):$ Suppose that $r m \in N-I N$ where $r \in R, m \in M$. If $r \notin[N+J(M): M]$, so either $[N: r]=N+J(M)$ or $[N: r]=[I N: r]$. Since $r m \notin I N$, so $m \notin[I N: r]$. But $r m \in N$, so $m \in[N: r]$. Then $[N: r]=N+J(M)$. Therefore $m \in N+J(M)$. Thus $N$ is an $I$-nearly prime submodule of $M$.

Proposition (1.9): Suppose that $M_{1}$ be an $R_{1}$-module and $M_{2}$ be an $R_{2}$-module. Then we have :

(1) If $N_{1}$ is an $I_{1}$ - nearly prime submodule of $M_{1}$ such that $I N_{1} \times M_{2} \subseteq I\left(N_{1} \times M_{2}\right)$ and $J\left(M_{1}\right) \times$ $M_{2} \subseteq J\left(M_{1} \times M_{2}\right)$, then $N_{1} \times M_{2}$ is an $I$ - nearly prime in $M_{1} \times M_{2}$.

(2): If $N_{2}$ is an $I_{2}$ - nearly prime in $M_{2}$ such that $I N_{2} \times M_{1} \subseteq I\left(N_{2} \times M_{1}\right)$ and $J\left(M_{2}\right) \times M_{1} \subseteq$ $J\left(M_{2} \times M_{1}\right)$, then $M_{1} \times N_{2}$ is an $I$ - nearly prime in $M_{1} \times M_{2}$. 
Proof: (1): Suppose that $(a, b) \in R_{1} \times R_{2}$ and $\left(m_{1}, m_{2}\right) \in M$ with $(a, b)\left(m_{1}, m_{2}\right)=\left(a m_{1}, b m_{2}\right) \in$ $N_{1} \times M_{2}-I\left(N_{1} \times M_{2}\right)$, and $N_{1} \times M_{2}-I\left(N_{1} \times M_{2}\right) \subseteq N_{1} \times M_{2}-I N_{1} \times M_{2}=\left(N_{1}-I N_{1}\right) \times M_{2}$. We have $a m_{1} \in N_{1}-I N_{1}$ but $N_{1}$ is an $I_{1}$ - nearly prime in $M_{1}$. So $a \in\left[N_{1}+J\left(M_{1}\right): M_{1}\right]$ or $m_{1} \in N_{1}+$ $J\left(M_{1}\right)$. So $(a, b) \in\left[N_{1}+J\left(M_{1}\right): M_{1}\right] \times R_{2}=\left[\left(N_{1}+J\left(M_{1}\right)\right) \times M_{2}:_{R_{1} \times R_{1}} M_{1} \times M_{2}\right]=\left[N_{1} \times M_{2}+\right.$ $\left.J\left(M_{1}\right) \times M_{2}:_{R_{1} \times R_{1}} M_{1} \times M_{2}\right] \subseteq\left[N_{1} \times M_{2}+J\left(M_{1} \times M_{2}\right):_{R_{1} \times R_{1}} M_{1} \times M_{2}\right] \quad$ or $\quad\left(m_{1}, m_{2}\right) \in\left[N_{1}+\right.$ $\left.J\left(M_{1}\right)\right] \times M_{2}=N_{1} \times M_{2}+J\left(M_{1}\right) \times M_{2} \subseteq N_{1} \times M_{2}+J\left(M_{1} \times M_{2}\right)$. Hence $N_{1} \times M_{2}$ is an $I-$ nearly prime submodule of $M_{1} \times M_{2}$.

The proof of (2) is similar to proof (1).

Proposition (1.10): Let $I_{1}$ and $I_{2}$ be ideals of $R_{1}$ and $R_{2}$, respectively, with $I=I_{1} \times I_{2}$.

1. $N_{1} \times N_{2}$ is an $I$-nearly prime in $M_{1} \times M_{2}$ where $I_{i} N_{i}=N_{i}$ for $i=1,2$.

2. If $N_{1}$ is a prime in $M_{1}$, then $N_{1} \times M_{2}$ is an $I$-nearly prime in $M_{1} \times M_{2}$.

3. If $N_{1}$ is an $I_{1}$ - nearly prime in $M_{1}$ with $I_{2} M_{2}=M_{2}$, then $N_{1} \times M_{2}$ is an $I$-nearly prime in $M_{1} \times M_{2}$.

4. If $N_{2}$ is a prime in $M_{2}$, then $M_{1} \times N_{2}$ is an $I$-nearly prime in $M_{1} \times M_{2}$.

5. If $N_{2}$ is an $I_{2}$ - nearly prime in $M_{2}$ with $I_{1} M_{1}=M_{1}$, then $M_{1} \times N_{2}$.

Proof (1): Since $I_{1} N_{1}=N_{1}$ and $I_{2} N_{2}=N_{2}$. Then $I_{1} N_{1} \times I_{2} N_{2}=\left(I_{1} \times I_{2}\right)\left(N_{1} \times N_{2}\right)=I\left(N_{1} \times N_{2}\right)=$ $N_{1} \times N_{2}$. So $N_{1} \times N_{2}-I\left(N_{1} \times N_{2}\right)=\emptyset$. Thus there is nothing to prove.

2. Let $N_{1}$ is a prime in $M_{1}$. Then $N_{1} \times M_{2}$ is a prime in $M_{1} \times M_{2}$, [9] and hence $I$ - nearly prime in $M_{1} \times M_{2}$.

3. Let $N_{1}$ is an $I_{1}$ - nearly prime in $M_{1}$ and $I_{2} M_{2}=M_{2}$. Suppose that $\left(r_{1}, r_{2}\right) \in R$ and $\left(m_{1}, m_{2}\right) \in$ $M$ with $\left(r_{1}, r_{2}\right)\left(m_{1}, m_{2}\right)=\left(r_{1} m_{1}, r_{2} m_{2}\right) \in N_{1} \times M_{2}-I\left(N_{1} \times M_{2}\right)=N_{1} \times M_{2}-\left(I_{1} \times I_{2}\right)\left(N_{1} \times\right.$ $\left.M_{2}\right)=\left(N_{1} \times M_{2}-\left(I_{1} N_{1} \times I_{2} M_{2}\right)=\left(N_{1} \times M_{2}-\left(I_{1} N_{1} \times M_{2}\right)=\left(N_{1}-I N_{1}\right) \times M_{2}\right.\right.$. Then $r_{1} m_{1} \in$ $N_{1}-I N_{1}$ and $N_{1}$ is an $I_{1}$ - nearly prime submodule of $M_{1}$, so $r_{1} \in\left[N_{1}+J\left(M_{1}\right): M_{1}\right]$ or $m_{1} \in N_{1}+$ $J\left(M_{1}\right)$.

Therefore $\left(r_{1}, r_{2}\right) \in\left[N_{1}+J\left(M_{1}\right): M_{1}\right] \times R_{2}=\left[\left(N_{1}+J\left(M_{1}\right)\right) \times M_{2}:_{R_{1} \times R_{1}} M_{1} \times M_{2}\right]=\left[N_{1} \times M_{2}+\right.$ $\left.J\left(M_{1}\right) \times M_{2}:_{R_{1} \times R_{1}} M_{1} \times M_{2}\right] \subseteq\left[N_{1} \times M_{2}+J\left(M_{1} \times M_{2}\right):_{R_{1} \times R_{1}} M_{1} \times M_{2}\right]$ or $\left(m_{1}, m_{2}\right) \in\left[N_{1}+\right.$ $\left.J\left(M_{1}\right)\right] \times M_{2}=N_{1} \times M_{2}+J\left(M_{1}\right) \times M_{2} \subseteq N_{1} \times M_{2}+J\left(M_{1} \times M_{2}\right)$. So $N_{1} \times M_{2}$ is an $I-$ nearly prime in $M_{1} \times M_{2}$.

The proofs of (4) and (5) are similar to parts (2), (3), respectively.

Proposition( 1.11): Let $M$ be an $R$-module and let $N$ be a proper submodule of $M$ such that $N / I N+$ $J(M / I N)=N+J(M) / I N$. Then $N$ is an $I$-nearly prime in $M$ if and only if $N / I N$ is 0 -nearly prime in $M / I N$.

Proof: Let $N$ be an $I$-nearly prime in $M$. Suppose that $0 \neq a x+I N=a(x+I N) \in N / I N$ in $M / I N$ where $a \in R, x \in M$. Then $a x \in N-I N$. Since $N$ is an $I$-nearly prime submodule of $M$, so either $x \in N+J(M) \quad$ or $a \in[N+J(M): M]=[[N+J(M)] / I N: M / I N]$. Therefore $\quad x+I N \in[N+$ $J(M)] / I N=N / I N+J(M / I N)$ or $a \in[N / I N+J(M / I N): M / I N]$. Hence $N / I N$ is 0 - nearly prime in $M / I N$.

Conversely, let $N / I N$ is an 0 -nearly prime in $M / I N$. Assume that $a \in R, x \in M$ with $a x \in N-$ $I N$. So $0 \neq a(x+I N)=a x+I N \in N / I N$. But $N / I N$ is an 0 -nearly prime in $M / I N$. Thus $x+$ $I N \in N / I N+J(M / I N)=[N+J(M)] / I N \quad$ or $\quad a \in[N / I N+J(M / I N): M / I N]=[[N+$ $J(M)] / I N: M / I N]$ and so $x \in N+J(M)$ or $a \in[N+J(M): M]$. Hence $N$ is an 0 -nearly prime.

Theorem (1.12): If $M$ is an $R$-module and $I$ is an ideal of $R$, then the following statements are equivalent.

1 - $I M$ is an $I$-nearly prime submodule $M$;

2- For $x \in[M \backslash(I M+J(M))] ;[I M: x]=[I(I M): x] \cup[I M+J(M): M]$;

3- For $x \in[M \backslash(I M+J(M))]$, $[I M: x]=[I(I M): x]$ or $[I M: x]=[I M+J(M): M]$.

4- If $J K \subsetneq I M-I(I M)$, then $J \subsetneq[I M+J(M): M]$ or $K \subsetneq I M+J(M)$ for each an ideal $J$ of $\mathrm{R}$ and submodule $K$ of $M$.

Proof : (1) $\rightarrow$ (2): Suppose that $x \in M-I M, r \in[I M: x]$. So $r x \in I M$. If $r x \notin I(I M)$, but $I M$ is an $I$ -nearly prime and $x \notin I M+J(M)$, so $r \in[I M+J(M): M]$. If $r x \in I(I M)$, so $r \in[I(I M): x]$. Thus, $[I M: x] \subsetneq[I M+J(M): M] \cup[I I M: x]$. On the other hand $I(I M) \subsetneq I M$, so $[I(I M): x] \cup[I M+$ $J(M): M] \subsetneq[I M: x]$.

$(2) \rightarrow(3)$ : It follows directly by the fact that if an ideal is a union of two ideals, then it is equal to one of them. 
(3) $\rightarrow$ (4): Suppose that $J K \subsetneq I M$. Let $J \nsubseteq[I M+J(M): M]$ and $K \nsubseteq I M+J(M)$. Ausssme that $x \in$ $K$. If $x \notin I M+J(M)$. So $J x \subsetneq I M$ and hence $J \subsetneq[I M: x]$. But $J \nsubseteq[I M+J(M): M]$, so $J \subsetneq[I M: x]=$ $[I(I M): x]$. Thus, $x J \subsetneq I(I M)$, so $K J \subsetneq I(I M)$. Suppose that $x \in I M$. Let $m \in K-I M$. Then $(x+$ $m) \in K-I M$. So $(x+m) J \subsetneq I(I M)$. Let $r \in J$. Then $x=(x+m) r-m r \in I(I M)$. So $x J \subsetneq I(I M)$. Thus $J K \subsetneq I(I M)$. (4) $\rightarrow$ (1): By theorem (1.4).

\section{Proposition (1.13):}

1- Let $N_{1}$ and $N_{2}$ are two submodules of the $R$-0modules $M_{1}, M_{2}$, respectively. If $N_{1} \oplus N_{2}$ is an $I$ nearly prime and small submodule of $M=M_{1} \oplus M_{2}$ such that $J\left(M_{1} \oplus M_{2}\right) \subseteq\left[J\left(M_{1}\right) \oplus M_{2}\right]$ and $J\left(M_{1} \oplus M_{2}\right) \subseteq\left[M_{1} \oplus J\left(M_{2}\right)\right]$, then $N_{1}$ and $N_{2}$ are $I$ - nearly prime in $M_{1}, M_{2}$ respectively.

2- Let $N$ be a small submodule of an $R$-module $M_{1}$ and $M_{2}$ be any two modules with $J\left(M_{1}\right) \oplus M_{2}$ is small in $M$. If $N$ is an $I$-nearly prime, then $N \oplus M_{2}$ is an I-nearly primes submodule of $M_{1} \oplus M_{2}$.

Proof. (1). Let $a m_{1} \in N_{1}-I N_{1}$ where $a \in R, m_{1} \in M_{1}$. Then $a\left(m_{1}, 0\right) \in\left(N_{1} \oplus N_{2}\right)-I\left(N_{1} \oplus N_{2}\right)$. Since $\left(N_{1} \oplus N_{2}\right)$ is an $I$-nearly prime and small, then either $\left(m_{1}, 0\right) \in\left(N_{1} \oplus N_{2}\right)+J(M)=J\left(M_{1} \oplus\right.$ $\left.M_{2}\right)=J\left(M_{1}\right) \oplus J\left(M_{2}\right),[10]$ and so $m_{1} \in J\left(M_{1}\right) \subseteq N_{1}+J\left(M_{1}\right)$ or a $\in\left[\left(N_{1} \oplus N_{2}\right)+J\left(M_{1} \oplus\right.\right.$ $\left.\left.M_{2}\right): M_{1} \oplus M_{2}\right]=\left[J\left(M_{1} \oplus M_{2}\right): M_{1} \oplus M_{2}\right] \subseteq\left[J\left(M_{1}\right) \oplus M_{2}: M_{1} \oplus M_{2}\right]$ and so a $\in\left[J\left(M_{1}\right): M_{1}\right]$ $\subseteq\left[N_{1}+J\left(M_{1}\right): M_{1}\right]$. It follows that either $m_{1} \in N_{1}+J\left(M_{1}\right)$ or a $\in\left[N_{1}+J\left(M_{1}\right): M_{1}\right]$. Hence $N_{1}$ is an $I$-nearly prime in $M_{1}$.

By a similar proof, $N_{2}$ is an $I$-nearly prime in $M_{2}$.

(2). Let $a\left(m_{1}, m_{2}\right) \in\left(N \oplus M_{2}\right)-I\left(N \oplus M_{2}\right)$, where $a \in R,\left(m_{1}, m_{2}\right) \in M$. Then $a m_{1} \in N-I N$. Since $N$ is an $I$-nearly prime and small in $M_{1}$, then either $m_{1} \in N+J\left(M_{1}\right)=J\left(M_{1}\right)$ or a $\in$ $\left[N+J\left(M_{1}\right): M_{1}\right],[10]$. So that

If $m_{1} \in N+J\left(M_{1}\right)=J\left(M_{1}\right)$, then $\left(m_{1}, m_{2}\right) \in J\left(M_{1}\right) \oplus M_{2} \subseteq J\left(M_{1} \oplus M_{2}\right) \subseteq N \oplus M_{2}+J\left(M_{1} \oplus\right.$ $\left.M_{2}\right)$.

If a $\in\left[N+J\left(M_{1}\right): M_{1}\right]$ and since $N$ is small in $M_{1}$, then a $\in\left[J\left(M_{1}\right) \oplus M_{2}: M_{1} \oplus M_{2}\right]$. But $J\left(M_{1}\right) \oplus$ $M_{2}$ is small in $M_{2}$, so $\left[J\left(M_{1}\right) \oplus M_{2}: M_{1} \oplus M_{2}\right] \subseteq\left[J\left(M_{1} \oplus M_{2}\right): M_{1} \oplus M_{2}\right] \subseteq\left[N \oplus M_{2}+J\left(M_{1} \oplus\right.\right.$ $\left.\left.M_{2}\right): M_{1} \oplus M_{2}\right]$, so $N \oplus M_{2}$ is an $I$-nearly prime in $M_{1} \oplus M_{2}$.

Corollary (1.14):

1-If $\quad N_{1} \oplus N_{2}$ is an $I$-nearly prime of a hollow module $M_{1} \oplus M_{2}$ with $J\left(M_{1} \oplus M_{2}\right) \subsetneq \quad\left[J\left(M_{1}\right) \oplus\right.$ $\left.M_{2}\right]$ and $J\left(M_{1} \oplus M_{2}\right) \subsetneq\left[M_{1} \oplus J\left(M_{2}\right)\right]$, then $N_{1}$ and $N_{2}$ are $I$-nearly primes in $M_{1}, M_{2}$ respectively.

2- If is $N$ an $I$-nearly prime submodule of a hollow $R$-module $M_{1}, M_{2}$ is any module such that $M_{1} \oplus M_{2}$ be a hollow $R$-module, then $N \oplus M_{2}$ is an $I$-nearly prime submodule of $M_{1} \oplus M_{2}$.

Proof: (1): Since $M_{1} \oplus M_{2}$ is a hollow , so all submodules are small , [11]. Therefore the result follows $(1.13,1)$.

(2): Since $M_{1}$ and $M_{2}$ are hollow modules, so every submodule of them is small, [11]. Therefore the result follows $(1.13,2)$.

\section{References}

1. Lu, C. 1984. Prime submodule of modules, Comment. Math., Univ. St Paul, 33(1): 61-69.

2. Abdul-Alkalik, A. J. 2017. Some Generalizations of prime submodules, Ph D. Thesis, University of Al-Mustansiriyah.

3. Akray, I. and Hussein, H. S. 2017. I-prime submodules, Acta Mathematica Academiae Paedagogicae, 33: 165-173.

4. Akray, I. and Hussein, H. S. 2016. I-primary submodules, Math. AC, 1(7).

5. Kasch, F. 1982. Modules and Rings, Academic Press, London.

6. Hirano, Y. and Mogani, I. 1986. On restricted anti-hopfian modules, Math. J. Okayama Universtiy, 28: $119-131$.

7. 7.Larsen, M. D. and McCarlthy, P. J. 1971. Multiplicative theory of ideals, Academic Press. New York.

8. Atiyah, M. F. and MacDonald, I. G. 1969. Introduction to Commutative Algebra, Oxford University Press. New York.

9. Khaksari, A. 2011. $\varphi$-prime submodules. International journal of algebra, 5(29): 1443-1449.

10. Hirano, Y. and Mogani, I. 1986. On restricted anti-hopfian modules, Math. J. Okayama Universtiy, (28): 119-131.

11. Dung, N. V., Huynh D. V., Smith, P. F., and Wishbauer, R. 1994. Extending modules, Pitman Reseaech Notes in Mathematics Series Longman Harlow. 